Artículo

\title{
Zonificación edafoclimática del cultivo de cacao en el estado Chiapas
}

\author{
Gicli Manuel Suárez Venero ${ }^{1}$ \\ Carlos Hugo Avendaño Arrazate ${ }^{2}$ \\ Manuel Antonio Hernández Ramos ${ }^{3}$ \\ Luís Alfredo Rodríguez Larramendi ${ }^{38}$ \\ Paulina Estrada de los Santos ${ }^{4}$ \\ Miguel Ángel Salas Marina ${ }^{3}$ \\ ${ }^{1}$ Universidad de Guantánamo. Avenida Che Guevara km 1.5, Carretera a Jamaica, Guantánamo, Cuba. \\ CP. 95100. (gicli@cug.co.cu). ${ }^{2}$ Campo Experimental Rosario Izapa-INIFAP. Carretera Tapachula- \\ Cacahoatán, Tuxtla Chico km 18, Chiapas, México. CP. 30870. (avendano.carlos@inifap.gob.mx). \\ ${ }^{3}$ Universidad de Ciencias y Artes de Chiapas-Facultad de Ingeniería, Sede Villa Corzo, Chiapas, México. \\ (manuel.hernández@unicach.mx; miguel.salas@unicach.mx). ${ }^{4}$ Escuela Nacional de Ciencias Biológicas- \\ Instituto Politécnico Nacional. México. CP. 07738. (pestradadelossantos@ gmail.com).
}

${ }^{\S}$ Autor para correspondencia: alfredo.rodriguez@unicach.mx.

\section{Resumen}

El estado de Chiapas es el segundo productor de cacao en México; sin embargo, los rendimientos agrícolas son bajos y en muchas regiones existen limitantes relacionadas con factores edafoclimáticos. Por ello, la investigación tuvo como objetivo identificar zonas edafoclimáticas que respondan a los requerimientos del cacao, en función de alcanzar un mayor rendimiento en el estado de Chiapas. La metodología utilizada se fundamentó y diseñó como una forma simplificada de la metodología general de la FAO y a partir de las propuestas metodológicas realizadas en los cultivos de café y cacao en otros países, con adecuaciones específicas a las condiciones particulares e información existente de Chiapas. Se determinaron las condiciones edafoclimáticas que caracterizan cuatro categorías de zonificación (óptima, medianamente óptima, aceptable y no apta) representadas en mapas a escala 1:100 000. Se identificó una superficie total de 1549804.4 ha con diferentes potenciales productivos, que representa $20.08 \%$ la superficie total del estado y distribuida en 59 municipios. De ellos 34 no tienen antecedentes del cultivo, lo que indica las posibilidades de explorar nuevas áreas para el mismo. Las temperaturas extremas (bajas y altas) y las propiedades físicas del suelo constituyeron los principales factores limitantes para el desarrollo del cacao y ocupan la mayor superficie no apta del estado. Desde el punto de vista práctico, los resultados constituyen una herramienta para la toma de decisiones en una amplia escala para el establecimiento del cultivo del cacao en Chiapas.

Palabras clave: Theobroma cacao L., clima, municipios, suelos, zonas.

Recibido: marzo de 2021

Aceptado: junio de 2021 


\section{Introducción}

El cacao (Theobroma cacao L.), es una planta que se cultiva en varios países del mundo por sus cualidades alimenticias y medicinales (Mcfadden, 2008). Sin embargo, a pesar de dicha importancia, los niveles de producción aún no satisfacen las demandas del producto. En México, en los últimos 10 años se ha observado una tendencia decreciente en la producción. La misma se concentra en Tabasco, Oaxaca y Chiapas. Este último ocupa el segundo lugar en producción después de Tabasco, con un promedio de $9707 \mathrm{t}$ y un rendimiento de $0.47 \mathrm{t} \mathrm{ha}^{-1}$ (SIAP, 2018).

Los factores ambientales, tecnológicos, económicos y sociales han influido en estos resultados y se ven agravados por problemas fitosanitarios como las enfermedades que destruyen plantaciones enteras (Hernández-Gómez et al., 2015). Desde el punto de vista ambiental, los cambios que ocurren con los patrones del clima y las transformaciones de las propiedades de los suelos hacen posible que muchas áreas pierdan las condiciones para el desarrollo del cacao en correspondencia con sus requerimientos edafoclimáticos. Esto trae consigo falta de compatibilidad entre las condiciones edafoclimáticas y las exigencias del cacao, como consecuencia de un uso inadecuado del suelo. Tal situación constituye una problemática a tener en cuenta en el diseño de las investigaciones para aumentar los rendimientos actuales del cacao (Suárez et al., 2015).

Particularmente en Chiapas, las regiones de tradición en el desarrollo del cultivo es en la parte Norte, Centro, Soconusco y Selva-Norte. Estas plantaciones se han desarrollado con criterios de la cultura tradicional y sin criterios de estudios de zonificación que validen científicamente las condiciones de estas superficies y que permitan la planificación adecuada de la tierra. Por lo anterior, es posible encontrar en la actualidad zonas con aptitudes para el desarrollo del cultivo que no se explotan, o zonas donde se está desarrollando el cultivo por mucho tiempo con pérdidas de sus aptitudes para el mismo. Esta última condición significa menores posibilidades de obtener el rendimiento potencial de acuerdo con las características de los agroecosistemas.

Para solucionar esos problemas, se han realizado diferentes estudios para la planificación del uso de las tierras por medio de la zonificación agroecológica (ZAE) y la zonificación edafoclimática (ZEC) de los cultivos (FAO, 1997). En estos temas, los trabajos más significativos que se han realizado en México son los de Puebla et al. (1991); Villa et al. (2001); Pérez y Geissert (2006); Martínez et al. (2007); Rivera et al. (2012); Hernández et al. (2014); González et al. (2015); Espinosa-García et al. (2015). El objetivo de la investigación fue ubicar zonas edafoclimáticas que respondan a los requerimientos del cultivo del cacao, en función de alcanzar mayor rendimiento y lograr un proceso de tomas de decisiones más efectivas en el estado de Chiapas.

\section{Materiales y métodos}

\section{Ubicación y selección del área de estudio}

La investigación se desarrolló en el estado de Chiapas, México (Figura 1). Este se localiza al sureste de México; colinda al norte con el estado de Tabasco, al oeste con Veracruz y Oaxaca, al sur con el Océano Pacífico y al este con la República de Guatemala. Al norte $17^{\circ}$ 59', al sur $14^{\circ}$ $32^{\prime}$ de latitud norte; al este $90^{\circ} 22^{\prime}$, al oeste $94^{\circ} 14^{\prime}$ de longitud oeste. Posee una superficie total de $77148.263 \mathrm{~km}^{2}$, la cual representa 3.8\% del territorio nacional (Gobierno de Chiapas, 2019). 


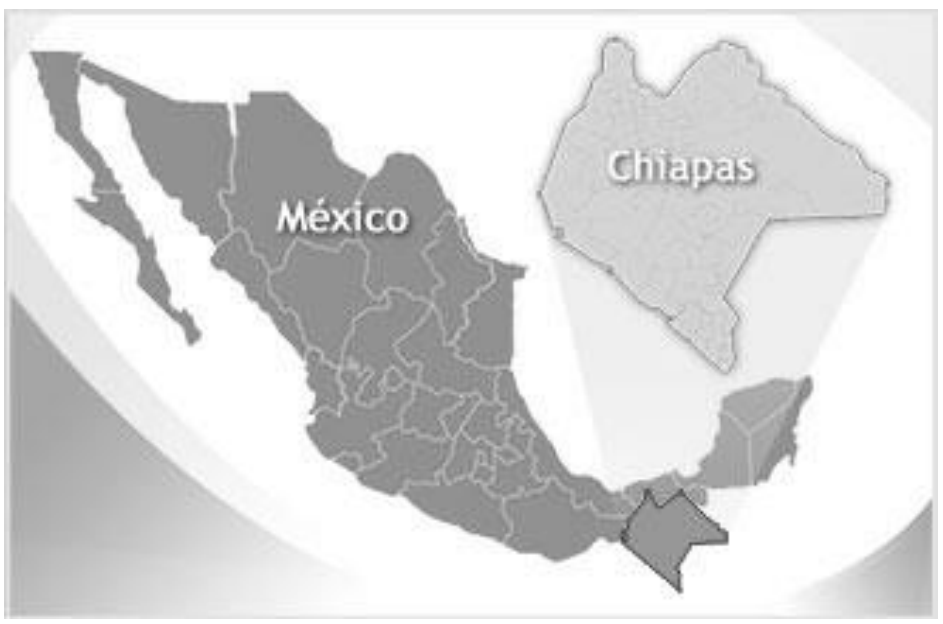

Figura 1. Localización del estado de Chiapas.

\section{Diseño metodológico}

El esquema general de la investigación se elaboró como una forma simplificada de la metodología general de la FAO (1997) y a partir de las propuestas metodológica realizadas por Soto et al. (2001) para el cultivo del café y Suárez et al. (2015) para el cultivo del cacao, adecuada específicamente a las condiciones particulares del estado de Chiapas, nivel de información y escala de trabajo.

Para ello se consideraron las precipitaciones anuales $(\mathrm{mm})$, temperatura media anual $\left({ }^{\circ} \mathrm{C}\right) \mathrm{y}$ grupos de suelos con sus propiedades naturales. En este contexto, se consideraron las bases para la zonificación edafoclimática para el cacao según Suárez et al. (2013) determinada por medio del criterio de expertos, para lo cual se aplicó la metodología Delphi descrita por Linstone y Turrof (1975).

Según el esquema metodológico empleado, se realizó una caracterización edafoclimática del estado y se obtuvo la representación de las características de clima y suelo con diferentes aptitudes para el desarrollo del cultivo del cacao. A partir de las combinaciones de los mapas temáticos, se elaboró el mapa resultante de la zonificación edafoclimática del cacao para el estado de Chiapas a escala 1:100 000, según los criterios de las bases de la zonificación en cuatro categorías: óptima, medianamente óptimas, aceptables y no aptas.

La delimitación de estas zonas se generó con el uso del ArcGIS 9.3 como sistema de información geográfica (SIG) que facilitó la manipulación y sobreposición de capas de información temática de relieve, clima y suelo.

\section{Base de datos edafoclimáticos}

Para el caso específico del clima, se recopiló la información existente de precipitaciones anuales y las temperaturas mínimas y máximas de 30 años (1986-2016) de la base de datos de CONAGUA (2018). La misma fue digitalizada y representadas según los criterios definidos en las bases de la zonificación para el cultivo. 
La base de información de suelos utilizada fue la existente en el Instituto Nacional de Estadística y Geografía (INEGI) del estado. En esta se encuentra la información geoespacial que muestra la distribución de los principales grupos de suelos en el territorio nacional, así como los atributos físicos, químicos y sus limitantes, de acuerdo con la base referencial mundial del recurso suelo de la FAO (IUSS, WRB, 2015). En esta misma fuente se obtuvo además la información básica del relieve utilizando información topográfica proveniente de un modelo de elevación digital (DEM) que sirvió para delimitar los niveles altitudinales del territorio.

\section{Resultados y discusión}

Caracterización climática del estado de Chiapas en función de la zonificación edafoclimática del cacao para evaluar las disponibilidades o limitantes climáticas de una región determinada, es imprescindible caracterizar en términos cuantitativos el comportamiento de los diferentes elementos del clima. De acuerdo con la clasificación de Köppen modificado por García (1973), el clima predominante en la región de Chiapas es del tipo Af (cálido húmedo con lluvia todo el año) y Am (cálido húmedo con lluvia en verano).

\section{Precipitaciones}

La región norte del estado presenta lluvias todo el año, en el resto de la entidad abundan las lluvias en verano. La precipitación total anual varía, dependiendo de la región, entre 1200 y 4 $000 \mathrm{~mm}$, así como grandes extensiones con una estación lluviosa (mayo-octubre) y una seca (noviembre-abril) perfectamente definidas (Figura 2).

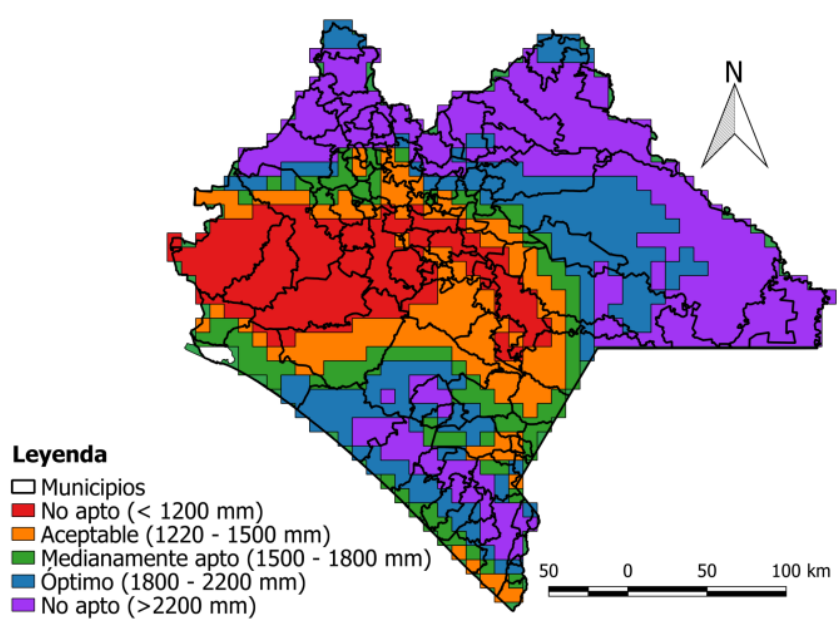

Figura 2. Mapa que representa la precipitación media anual según criterios de aptitud para el cultivo del cacao en el estado de Chiapas, México.

En $21.56 \%$ del territorio ocurrieron precipitaciones que oscilaron entre $1800 \mathrm{~mm}$ y más de 2200 $\mathrm{mm}$, con un promedio anual de $2100 \mathrm{~mm}$, valores extremos de precipitaciones adecuadas para el crecimiento y desarrollo del cultivo de cacao. No obstante, en la mayor parte de la superficie total del estado (56.87\%) se registró un nivel pluviométrico compatible para el normal crecimiento y desarrollo del cultivo del cacao (González, 2008). 
En la parte norte coincidente con la cordillera centroamericana y zonas de la sierra de Chiapas y Guatemala, se registraron precipitaciones extremas máximas con valores entre 3000 y $4000 \mathrm{~mm}$, lo que hace posible un exceso de humedad y un régimen pluviométrico que no cumplen las exigencias para el normal desarrollo del cultivo. Lo anterior, está relacionado con niveles altitudinales por encima de los $750 \mathrm{~m}$. Lo contrario se registró en la llanura costera del sur donde las precipitaciones medias anuales no superan los $800 \mathrm{~mm}$, creando condiciones de déficit de humedad para el cultivo.

\section{Temperatura}

La temperatura media anual, también varía dependiendo de la región, de $18{ }^{\circ} \mathrm{C}$ en los Altos de Chiapas, a $28{ }^{\circ} \mathrm{C}$ en la Llanura Costeña, con la temperatura promedio más alta de $30{ }^{\circ} \mathrm{C}$ y la mínima de $17.5{ }^{\circ} \mathrm{C}$ (Figura 3).

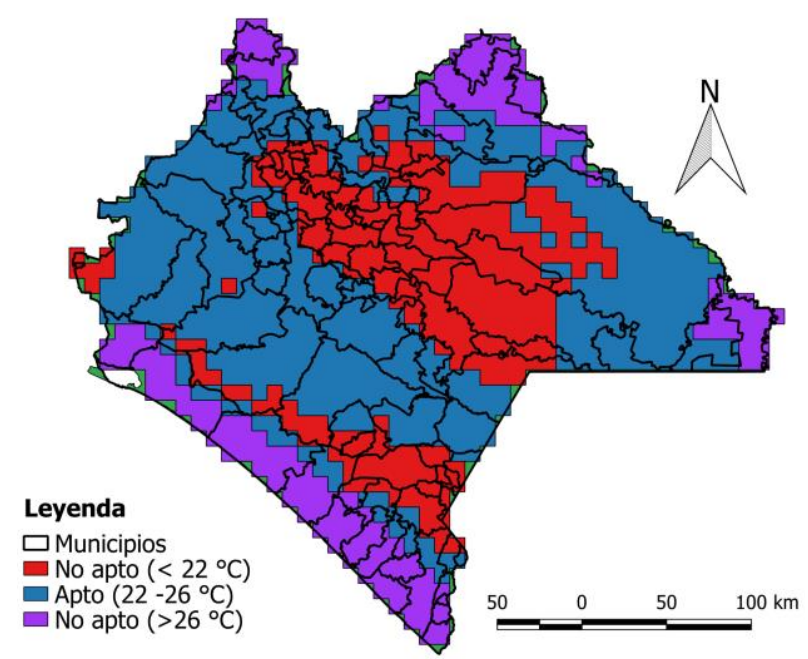

Figura 3. Mapa que representa la temperatura media según criterios de aptitud para el cultivo del cacao en el estado de Chiapas, México.

Posee zonas calientes con temperaturas medias superiores a $\operatorname{los} 23{ }^{\circ} \mathrm{C}$ y $\sin$ grandes oscilaciones térmicas y zonas semicálidas a una altitud entre 800 y $1550 \mathrm{~m}$, con temperaturas constantes de $20{ }^{\circ} \mathrm{C}$. También existen zonas templadas popularmente llamadas frías, situadas por encima de los $1500 \mathrm{msnm}$, en las cuales la temperatura presenta oscilaciones entre 12 y $15^{\circ} \mathrm{C}$ y la cumbre del volcán de Tacaná que tiene un clima frío, ya que está por encima de los 4000 msnm.

En $45.2 \%$ de la superficie total del territorio se registraron temperaturas entre $22{ }^{\circ} \mathrm{C}$ y $26{ }^{\circ} \mathrm{C}$, con un promedio anual de $24.8{ }^{\circ} \mathrm{C}$. Estos valores se corresponden con el comportamiento de esta variable en las principales zonas de producción de cacao en el mundo. Por tanto, en esta superficie esta variable climática suple las exigencias del cultivo, pues el mismo requiere de una temperatura promedio mensual óptima de $25{ }^{\circ} \mathrm{C}$.

Al respecto, Gómez y Azócar (2002) informaron que el rango general de temperatura promedio mensual para el cultivo del cacao oscila entre de $15{ }^{\circ} \mathrm{C}$ y $30{ }^{\circ} \mathrm{C}$, con un valor promedio de 25.5 ${ }^{\circ} \mathrm{C}$. Alvim (1984) informó que la floración del cacao se produce a partir de los $21{ }^{\circ} \mathrm{C}$, con un 
óptimo de $25.5^{\circ} \mathrm{C}$. Por su parte, Ramos y Gómez (2004); Batista (2009), coinciden en que estos valores de temperatura son óptimos para asegurar un crecimiento regular del árbol, con abundante formación de flores y frutos, distribución adecuada de brotación de yemas y hojas nuevas durante el año.

Sin embargo, a pesar de lo anteriormente explicado, en la mayor parte del territorio (54.8\%), se registraron valores de temperaturas extremas para el cultivo ya sea por debajo de los $22{ }^{\circ} \mathrm{C}$ coincidente con alturas superiores a $850 \mathrm{~m}$ o por encima de $28{ }^{\circ} \mathrm{C}$ en las zonas más bajas del norte y sur del territorio, convirtiéndose en un factor limitante para la producción de cacao en el estado.

\section{Caracterización edáfica del estado de Chiapas en función de la zonificación del cultivo del cacao}

Para realizar la caracterización edáfica en este territorio en función de la zonificación edafoclimática del cacao, es importante reconocer los requerimientos edáficos del cultivo. En el cacao, por ser un cultivo perenne, la selección del suelo constituye la mayor responsabilidad en la etapa de su establecimiento y es fundamental para obtener alta productividad.

Espinal et al. (2005), informaron que el cacao requiere suelos con dos características opuestas en un mismo grupo: retención de humedad en periodo poco lluvioso, y a la vez buen drenaje en época lluviosa, además de ser suelos profundos. Estas características están determinadas por la naturaleza de estos, fundamentalmente por sus características físicas. En consecuencia, la selección de los grupos de suelos que respondan a dichas exigencias es de vital importancia para la determinación de las zonas para el desarrollo cacaotero.

De los 15 grupos de suelos clasificados por la IUSS, Working Group WRB (2015), en el estado de Chiapas predominan nueve grupos y de ellos solo cuatro suplen los requerimientos edáficos del cacao (Figura 4). Lo anterior está relacionado con las características climáticas y el relieve, pues los mismos determinan el proceso pedogenético de los suelos (Zavala-Cruz et al., 2014).

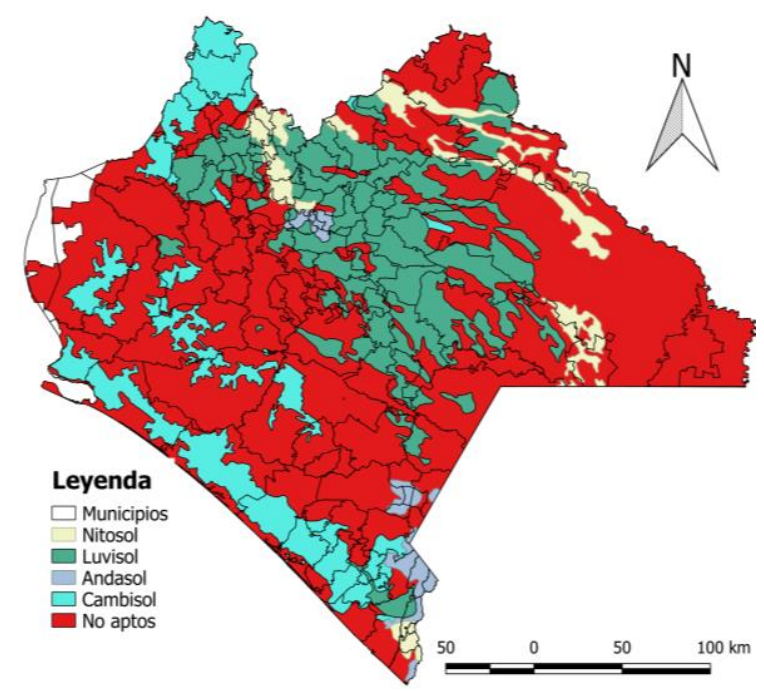

Figura 4. Mapa que representa la distribución de los suelos según requerimientos del cultivo del cacao en el estado de Chiapas, México. 
Los grupos de suelos existentes en Chiapas compatibles con las exigencias del cacao son: Cambisol, Luvisol, Nitosol y Andosol (Figura 4). Sus principales características según las exigencias del cultivo se describen a continuación.

Cambisol: incluye una superficie de 953037 ha que significa 12.35\% de la superficie total del estado. Por su pedogénesis, poseen buena estructura y profundidad, requerimientos imprescindibles para crecimiento del cultivo del cacao. Además, por lo general no suceden lavado de nutrientes importantes, pues dado su juventud y espesor atesoren muchos minerales alterables que se desprenden de su roca madre con los procesos de intemperismo.

Luvisol: consta de una superficie de 1525695 ha que significa 19.77\% de la superficie total del estado. Es el quinto grupo de suelos más importante por su extensión territorial en México y el primer grupo de suelos en Chiapas (INEGI, 2017).

La mayoría de los Luvisoles son referenciados como suelos fértiles y generalmente tiene alto grado de saturación de bases. Una buena parte de los Luvisoles en México son rojos (38.1\%), pobres en calcio y magnesio $(24.8 \%$ ) pero con alto contenido de Carbono Orgánico (más de $1 \%$ en los primeros $50 \mathrm{~cm}$ de profundidad). En este suelo predomina la presencia de arena, característica que proporciona drenaje rápido, característica beneficiosa para el cultivo del cacao. Investigaciones desarrolladas por Gómez-González et al. (2018) para el cultivo del café en Chiapas, caracterizaron este tipo de suelo con un horizonte B árgico, CIC mayor de $24 \mathrm{cmol}^{+} \mathrm{kg}^{-1}$ de arcilla y una saturación de bases mayor de $50 \%$.

Nitisol: engloba una superficie de 367891.9 ha que significa el $4.76 \%$ de la superficie total del estado. Poseen una alta estabilidad estructural y se consideran suelos fértiles a pesar de su bajo contenido en fósforo asimilable y su baja saturación en bases. Son profundos, estables y con propiedades físicas favorables para el cultivo del cacao (Working Group WRB, 2015).

Andosol: abarca una superficie de 118751.6 ha que significa $1.53 \%$ de la superficie total del estado. Poseen adecuada profundidad efectiva para el desarrollo del sistema radical del cacao. Son suelos generalmente fértiles, particularmente los Andosoles que se desarrollan sobre ceniza volcánicas intermedias o básicas y no expuestos a un lavado excesivo de sus nutrientes. Su principal limitación es la elevada capacidad de fijación de fosfatos causada por el aluminio activo; sin embargo, este efecto puede ser minimizado con la aplicación de materia orgánica y fertilizante fosfatado (Working Group WRB, 2015).

Cada una de estas unidades de suelo tiene asociaciones de procesos morfodinámicos, determinados por los efectos de la meteorización física y química del clima que tiene efectos en los procesos pedogenéticos primarios Working Group, WRB (2015). Por ello, en el territorio objeto de estudio, estos suelos cumplen diferentes regularidades en su distribución geográfica, lo cual determina el área que ocupan los mismos.

Según el esquema de zonalidad geográfica vertical de los suelos en este territorio, estos se ubican a distintas alturas con predominios de diferentes condiciones de clima que, unido con el relieve, la vegetación, el tiempo y el material de origen como agentes formadores de los suelos, pudieron haber influido en esta distribución (Hernández et al., 2006). 
Sin embargo, a los mismos tiempos estos pueden constituir los factores limitantes del entorno que limitan la productividad en muchos agroecosistemas, factores limitantes que se observan reflejados en aquellos grupos de suelos no seleccionado para el desarrollo del cultivo.

Los grupos de suelos existentes en el estado que no fueron seleccionados para el cacao (Acrisol, Litosol, Regosol, Solonchak, Vertisol), abarcan una superficie de 4749451.4 ha que significa $61.5 \%$ de la superficie total del estado y poseen los mayores factores limitantes de la agroproductividad que afectan el normal desarrollo del cultivo en el estado, comparados con los que fueron seleccionados.

En el caso particular de los Acrisoles, las principales limitantes para el cultivo es su fuerte acidez y baja saturación de bases provocada por su fuerte alteración. La pobreza en nutrientes minerales, la toxicidad por aluminio, la fuerte adsorción de fosfatos y la alta susceptibilidad a la erosión, son las principales restricciones a su uso.

Respecto a la toxicidad del aluminio, Hernández et al. (2006) indicaron que cuando la cantidad de este elemento en la solución del suelo alcanza concentraciones superiores a $1 \mathrm{cmol}^{+} \mathrm{kg}^{-1}$, las implicaciones directas sobre el crecimiento de las plantas por intoxicación son notables. Por otra parte, Vera et al. (2000) afirmaron que este catión puede limitar el desarrollo exitoso del cacao por sus efectos sobre la nutrición mineral y crecimiento radical de la planta. Las raíces se vuelven más gruesas y presentan puntos muertos. Si se acumula en las raíces impide la absorción y el traslado del calcio y el fósforo a la parte aérea, provocando deficiencias de estos elementos en la planta.

Los Litosoles, son un tipo de suelo que predomina en escarpas y afloramientos rocosos. Su espesor es menor a $10 \mathrm{~cm}$ y sostiene una vegetación baja, por lo tanto, su poca profundidad y alta pedregosidad limitan el desarrollo del cacao. En los Regosoles, la evolución del perfil es mínima como consecuencia de su juventud. Por ello, su escasa evolución le confiere características negativas para ser empleados en el cultivo del cacao por limitantes físicas y químicas.

Los suelos Solonchaks tienen como principal limitante su carácter salino. Se encuentran en regiones áridas o semiáridas. Presentan una capacidad de utilización muy reducida, solo para plantas tolerantes a la sal, aspecto no compatible con los requerimientos del cultivo. El tipo Vertisol posee alto contenido de minerales de arcilla expansiva y pesadas, entre ellos muchas montmorillonitas, que forman profundas grietas en las estaciones secas. Estos se vuelven muy duros en la estación seca y muy plásticos en la húmeda. Por lo tanto, aunque su fertilidad natural es alta, su composición textural pesada hace posible su incompatibilidad con las exigencias del cacao.

\section{Zonificación edafoclimática para el cacao en el estado de Chiapas, México}

Los resultados de la combinación entre las zonas climáticas y edáficas apropiadas a las exigencias del cultivo, considerando las bases de la zonificación definida para el cacao, originaron las zonas edafoclimáticas las cuales están representadas en la Figura 5 y su distribución en los municipios en el Cuadro 1. 


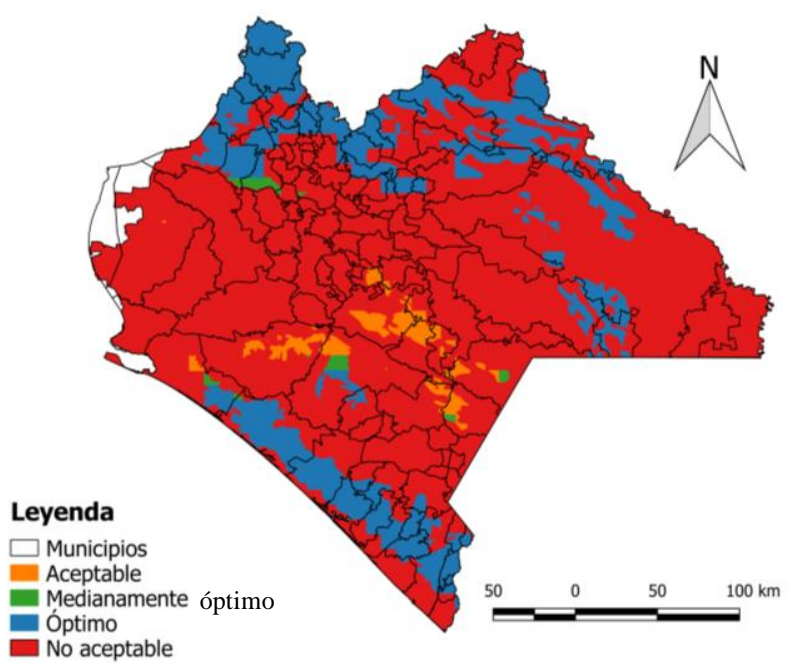

Figura 5. Mapa que representa la zonificación edafoclimática para el cultivo del cacao en el estado de Chiapas, México.

Cuadro 1. Municipios con superficies representadas por alguna categoría de zonificación edafoclimática para el cacao en el estado de Chiapas, México.

Municipios por categorías

con aptitudes para el Municipios

desarrollo del cacao

Zonas óptimas (48) A. Albino Corzo, Acacoyagua*, Acapetagua*, Amatán, Cacahoatán*, Chalchihuitán, Chapultenango, Chenalhó, Chilón, Escuintla*, F. de León*, F. Hidalgo*, Huehuetán*, Huitiupán, Huixtla*, Ixtapangajoya*, Ixtacomitán*, Juárez*, La Concordia, La Libertad, La Margaritas*, M. Tenejapa, Mapastepec ${ }^{*}$, Metapa*, Motozintla, Ocosingo, Ostuacán*, Palenque*, Pantheló, Pichucalco*, Pijijiapan, Reforma, Sabanilla, Salto de agua*, Simojovel, Sitalá, Solosuchiapa, Suchiate*,

Tapachula*, Técpatan*, Teopatán, Tila, Tonalá, Tumbalá, Tuxtla Chico*, Tuzantán*, Unión Juárez y V. Comatitlán*.

Zona medianamente Coatilla, Copainalá, Chicomuselo, F. Comalá, La Trinitaria, óptima (7) Técpatan* y Tonalá*

Zonas aceptables (11) Chicomuselo, F. Comalá, La Concordia, La Trinitaria y La Rosa, Socoltenango, Tzimol, Tonalá, Totolapa, V. Carranza y Villa Corzo

* = municipios con antecedentes en la producción de cacao en el estado Chiapas, México.

Zona óptima: comprende una superficie de 1349461.4 ha en el estado, que representa $17.5 \%$ de la superficie total en Chiapas. De los 118 municipios que componen el estado, esta categoría está representada en 35 municipios, siendo Tuxtla Chico, Metapa, Tuzantán, Yajalón, Iztapangajoya, Reforma y Juárez, los municipios con $100 \%$ de sus superficies representadas en esta categoría. Las plantaciones de cacao que se desarrollan en estas superficies pueden alcanzar un rendimiento mayor de $2 \mathrm{t} \mathrm{ha}^{-1}$ si el factor tecnológico no es limitante para la producción. 
Por otra parte, todos los municipios que actualmente cultivan el cacao se encuentran representados en esta categoría, lo cual corrobora las condiciones edafoclimáticas adecuadas para el cultivo en estos municipios y validan los resultados obtenidos en la presente investigación.

Zona Medianamente óptima: engloba una superficie de 40343 ha en el estado, que representa $0.52 \%$ de la superficie total en Chiapas. Se representan superficies pertenecientes a siete municipios, de ellos Técpatan es el único con antecedentes en el desarrollo del cultivo del cacao. Las plantaciones de cacao que se desarrollan en estas superficies pueden alcanzar un rendimiento entre 1 y $0.99 \mathrm{t} \mathrm{ha}^{-1}$ si se mantiene un nivel adecuado en el manejo del cultivo.

Zona aceptable: cubre una superficie de 160000 ha en el estado, que representa $2.07 \%$ de la superficie total en Chiapas. Se representan superficies pertenecientes a once municipios, ninguno de los cuales tiene antecedentes en el desarrollo del cultivo del cacao. Dentro de esta categoría, los municipios Chicomuselo, Frontera Comalá, La Trinitaria y Tonalá, comparten la misma con una u otra categoría identificada. Potencialmente las plantaciones de cacao que se desarrollen en estas superficies pueden alcanzar un rendimiento entre 0.5 y $0.99 \mathrm{t} \mathrm{ha}^{-1}$ con un manejo tecnológico adecuado.

Zona no apta: Abarca una superficie de 6165021.9 ha en el estado, que representa $69.91 \%$ de la superficie total en Chiapas. De los 118 municipios que componen el estado, esta categoría está representada en 59 municipios, ninguno de los cuales tienen antecedentes en el desarrollo del cultivo del cacao. Las principales limitantes de esta zona que restringen el desarrollo del cultivo son las temperaturas extremas (altas o bajas) motivadas por la altura sobre el nivel del mar y las propiedades físicas del suelo. Estas condiciones limitantes ocupan la mayor superficie en el estado (Figuras 3 y 4), por lo que el rendimiento potencial para esta zona es muy bajo, cuyos valores se planifican por debajo de los límites para que una plantación sea económicamente rentable.

En general, en el estado Chiapas existen 1549804.4 ha con características de clima y suelo adecuadas para el desarrollo del cultivo del cacao, lo cual significa $20.08 \%$ del territorio estatal con diferentes rendimientos y potencialidades. Los resultados descritos difieren de lo obtenido por Espinosa-García et al. (2015) quienes determinaron un total de 3691000 ha con potencialidades para producir cacao en el estado utilizando el programa SWAT como modelo hidrológico que se aplica a nivel de cuenca en nueve estados del Sursureste de México.

En la actualidad el cacao se cultiva en 25 municipios del estado, con la mayor superficie y municipios en la región del Soconusco que, por su clima tropical, es una de las regiones donde el cacao se cultiva desde tiempos ancestrales. Sin embargo, los resultados de la presente investigación permitieron distinguir las potencialidades edafoclimáticas para el establecimiento del cacao en 59 municipios con diferentes potenciales productivos. Ello indica la existencia de nuevas superficies con aptitudes para el cultivo que se ubican en 34 municipios sin antecedentes en su producción. Solo los municipios de Mazatán y Sunuapa con antecedentes del cultivo no están representados en las zonas edafoclimáticas con alguna categoría de aptitud.

Desde el punto de vista práctico, los resultados ponen a disposición de los productores y los tomadores de decisiones una herramienta útil sobre la ubicación del cacao en zonas edafoclimáticas adecuadas para incrementar los rendimientos actuales. Sin embargo, por ser 
amplia la escala de trabajo utilizada, estos no permiten a los decisores adoptar las medidas para el establecimiento del cultivo a nivel local, pero contribuye a planificar acciones y programas de trabajo a nivel municipal y estatal para la redistribución de las áreas de siembra.

La ubicación del cacao en zonas con adecuadas condiciones de clima y suelo constituye una de las estrategias agrícolas para impulsar la agricultura de precisión y contribuye a enfrentar los retos de los cambios que en la actualidad ocurren en algunos patrones del clima global y que afecta a la agricultura (Valdés y Vargas, 2011). En este contexto, las plantaciones de cacao son sitios con alta capacidad de captura de dióxido de carbono $\left(\mathrm{CO}_{2}\right)$ y liberación de oxígeno $\left(\mathrm{O}_{2}\right)$ por la gran cantidad de biomasa que produce y el hábito de crecimiento de este. Por lo que el establecimiento del cultivo en sitios donde se realice un crecimiento y desarrollo más eficiente contribuirá al enfrentamiento de los efectos del cambio climático.

La metodología empleada en la presente investigación ha permitido la identificación de zonas con diferentes aptitudes para el cultivo del cacao en el estado de Chiapas para colaborar con el proceso de toma de decisiones a amplia escala sobre su establecimiento y apoyo a los planes de desarrollo del cultivo. Por medio de ella, se han simplificado los fundamentos metodológicos utilizados por la FAO (1997) y metodologías empleadas en esta y otras regiones en el país.

Al respecto, la mayoría de los autores han utilizado los criterios edafoclimáticos para los estudios de la zonificación agroecológica de los cultivos, entre ellos se destaca la investigación realizada en los sistemas agroforestales en México por Pérez y Geissert (2006); Rivera et al. (2012) en el cultivo de la yuca (Manihot esculenta Crantz) en el estado de Tabasco, México. Sin embargo, no todas las variables edáficas y climáticas tienen el mismo peso en el momento de definir las zonas adecuadas para ubicar un cultivo, debido a la relación que existe entre ellas mismas y entre estas con las tecnologías aplicadas.

Se recomienda el uso de estos resultados para facilitar el diseño de políticas públicas que consideren el aumento de nuevas plantaciones o el reordenamiento de las áreas para cacao. Asimismo, dentro del proceso de toma de decisiones se deberá sobreponer esta información con el actual uso de la tierra para delimitar zonas de riesgos, zonas coincidentes y zonas que poseen usos infraestructurales.

\section{Conclusiones}

Se identificó una superficie de 1549804.4 ha con diferentes potenciales productivos para el desarrollo del cultivo del cacao, que representa $20.08 \%$ del territorio total del estado de Chiapas y ubicadas en superficies de 59 municipios, de ellos 34 no tienen antecedentes con el cultivo. Las temperaturas extremas (bajas y altas) relacionadas con el relieve y las propiedades físicas del suelo, constituyeron los principales factores limitantes para el desarrollo del cultivo de cacao en Chiapas y ocupan la mayor superficie con categoría no apta del estado.

Desde el punto de vista práctico, los resultados constituyen una herramienta para colaborar con el proceso de toma de decisiones a amplia escala sobre el establecimiento del cultivo del cacao como un cultivo de importancia económica para el estado de Chiapas. 


\section{Literatura citada}

Alvim, P. 1984. Influencia de Erithrina sobre algunos factores edáficos relacionados con la producción de cacao. Brasil: CEPEC. Informe técnico núm. 54.67 p.

Batista, L. 2009. Guía técnica. El cultivo del cacao en la república dominicana. Santo domingo, República Dominicana. CEDAF. 250 p.

CONAGUA. 2018. Comisión Nacional del Agua. Información climatológica del estado de Chiapas. http://smn.cna.gob.mx/es/climatologia/informacion-climatologica.

Espinal, C. F.; Martínez, H.; Beltrán, S. y Ortiz, L. 2005. La cadena del cacao en Colombia. Una mirada global de su estructura dinámica. Ministerio de Agricultura y Desarrollo Rural. Observatorio Agrocadenas. Colombia. 49 p.

Espinosa-García, J. A.; Uresti-Gil, J.; Vélez-Izquierdo, A.; Moctezuma-López, G.; InurretaAguirre, H. D. y Góngora-González, S. F. 2015. Productividad y rentabilidad potencial del cacao (Theobroma cacao L.) en el trópico mexicano. Mex. Cienc. Agríc. 6(5):1051-1063.

FAO. 1997. Boletín de suelos de la FAO Núm. 73. Food and agriculture organization of the United Nations. Roma, Italia 154 p.

García, E. 1973. Modificaciones del sistema de clasificación climática de Köppen para adaptarlo a las condiciones de la República Mexicana. $2^{\mathrm{a}}$ (Ed). Series libros 6, Instituto de Geografía-Universidad Nacional Autónoma de México (UNAM). México, DF. 246 p.

Gobierno de Chiapas. 2019. Conoce Chiapas. http://www.chiapas.gob.mx/conoce-chiapas/.

Gómez, A. y Azócar, A. 2002. Áreas potenciales para el desarrollo del cacao en el estado de Mérida. Agronomía Tropical. 52(4):403-425.

Gómez-González, R.; Palma-López, D. J.; Obrador-Olan, J. J. y Ruiz-Rosado, O. 2018. Densidad radical y tipos de suelos en los que se produce café (Coffea arabica L.) en Chiapas, México. Ecosistemas y Recursos Agropecuarios. 5(14):203-215.

González, F. 2008. Ecofisiología del cacao. Universidad Agraria de la Selva. tingo-maría. Perú http://Diplomado2007unas.blogspot.com/2008/01/ecofisiología-del-cacao.html.

González-Mancillas, R.; Juárez-López, J. F.; Aceves-Navarro, L. A.; Rivera-Hernández, B. y Guerrero-Peña, A. 2015. Zonificación edafoclimática para el cultivo de Jatropha curcas L. en Tabasco, México. Investigaciones geográficas boletín del instituto de geografía UNAM. 86:25-37. https://doi.org/10.14350/rig.39936.

Hernández, A.; Ascanio, M. O.; Morales, M.; Bojóquez, J. I. y García, N. E. 2006. Fundamentos sobre la formación del suelo, los cambios globales y su manejo. $1^{\text {a }}$ (Ed.). Universidad Autónoma de Nayarit. Nayarit, México. 255 p.

Hernández-Gómez, E.; Hernández-Morales, J.; Avendaño-Arrazate, C. H.; López-Guillen, G.; Garrido-Ramírez, E. R.; Romero-Nápoles, J. y Nava-Díaz, C. 2015. Factores socioeconómicos y parasitológicos que limitan la producción del cacao en Chiapas, México. Rev. Mex. Fitopatol. 33(2):232-246.

Hernández-Librado, V.; Martínez-Nayelli, A.; Vidal-Moctezuma, H.; López-Rocha, D. y Contreras-Roberto, G. 2014. Propuesta de un plan de desarrollo integral del guanábano (Annona muricata L.) en el estado de Veracruz México. Rev. Brasil. Fruticul. 36(esp. 1):94-101. https://doi.org/10.1590/S0100-29452014000500011.

Instituto Nacional de Estadística y Geografía. 2017. Anuario estadístico y geográfico de Chiapas. Gobierno del Estado de Chiapas. 739 p. https://www.datatur.sectur.gob.mx/ITxEF_ Docs/chis_anuario_pdF.pdf. 
IUSS, W. and Group W. 2015. World reference base for soil resources 2014, update 2015 International soil classification system for naming soils and creating legends for soil maps. World soil resources reports 106. FAO, Rome, Italy. 181 p.

Linstone, H. A. and Turrof, M. 1975. The Delphi method, techniques and applications. $1^{\text {st }}$ (Ed.). Addison Wesley Publishing. 620 p.

Martínez-Fonseca, J. L.; Tijerina-Chávez, L.; Arteaga-Ramírez, R.; Vázquez-Peña, M. A. y Becerril-Román, A. E. 2007. Determinación de zonas agroclimáticas para la producción de mango (Mangifera indica L. "Manila") en Veracruz, México. Investigaciones Geográficas. 63:17-35.

Mcfadden, C. 2008. Historia del chocolate. El chocolate como medicina. Avizora: Publicaciones. http://www.avizora.com/publicaciones/gastronomia/textos/0038_historia_chocolate.htm.

Pérez, E. and Geissert, D. 2006. Zonificación agroecológica de sistemas agroforestales: el caso café (Coffea arabica L.) palma camedor (Chamaedorea elegans Mart.). Interciencia. 31(8):556-562.

Puebla, A.; Aceves, L. A.; Ortiz, C. A.; Arteaga, R. y Villalpando, O. K. 1991. Zonificación agroecológica para el cacao (Theobroma cacao, L), en Tabasco. Agrociencia serie aguasuelo-clima. 2(2):89-106.

Ramos, C. G.; Gómez, A. y Ascencao, A. 2004. Caracteres morfológicos determinantes en el cacao (Theobroma cacao L.), del occidente de Venezuela. Agronomía Tropical. 54(1):4562. https://dialnet.unirioja.es/servlet/articulo?codigo $=7444156$.

Rivera-Hernández, B.; Aceves-Navarro, L. A.; Juárez-López, J.; Palma-López, D. J.; GonzálezMancillas, R. y González-Jiménez, V. 2012. Zonificación agroecológica y estimación del rendimiento potencial del cultivo de la yuca (Manihot esculenta Crantz) en el estado de Tabasco, México. Avances en Investigación Agropecuaria. 16(1):29-47.

SIAP. 2018. Intención de cosecha de cultivos perennes por estado en México 2018. Servicio de Información Agroalimentaria y Pesquera. México. http://infosiap.siap.gob.mx/opt/ agricultura/intension/Intencion_cosechaPerenne_estado2018.pdf.

Soto, F.; Tejeda, T.; Hernández, A. y Florido, R. 2001. Metodología para la zonificación agroecológica de (Coffea arabica, L) en Cuba. Cultivos Tropicales. 22(4):51-53.

Suárez, G. M.; Soto, F.; Garea, E.; Hernández, A.; Solano, O. y Florido, R. 2015. Zonificación edafoclimática de Theobroma cacao L. en el macizo montañoso nipe-sagua-baracoa. Investigación y Saberes. 4(1):57-68.

Suárez, G. M.; Florido, R.; Soto, F. y Caballero, A. 2013. Bases para la zonificación agroecológica en el cultivo del cacao (Theobroma cacao L.) por medio del criterio de expertos. Cultivos Tropicales. 34(2):30-37.

Valdés, N. y Vargas, D. 2011. Gases de efecto invernadero en la agricultura, un llamado a la acción. In: Ríos, H.; Vargas, D. y Funes, F. (Ed.). Innovación agroecológica, adaptación y mitigación del cambio climático. Mayabeque, Cuba. 15-23 p.

Vera, M.; Rosales, H. y Ureña, N. 2000. Caracterización físico-química de algunos suelos cacaoteros de la zona sur del lago de maracaibo, venezuela. Geográfica Venezolana. 41(2):257-270.

Villa, M.; Inzunza, M. y Catalán, E. 2001. Zonificación agroecológica de hortalizas involucrando grados de riesgo. Terra Latinoam. 19(1):1-7.

Zavala-Cruz, J.; Salgado-García, S.; Marín-Aguilar, A.; Palma-López, D.; Castelán-Estrada, M. y Ramos-Reyes, R. 2014. Transecto de suelos en terrazas con plantaciones de cítricos en Tabasco. Ecosistemas y Recursos Agropecuarios. 1(2):123-137. 